\title{
Pas si simple de refuser l'héritage! Les exemples d'André Gide et de Sylvie Germain
}

\section{Alain Goulet}

\section{(2) OpenEdition}

1 Journals

\section{Édition électronique}

URL : http://journals.openedition.org/studifrancesi/1045

DOI : 10.4000/studifrancesi. 1045

ISSN : 2421-5856

Éditeur

Rosenberg \& Sellier

\section{Édition imprimée}

Date de publication : 1 novembre 2014

Pagination : $543-553$

ISSN : 0039-2944

\section{Référence électronique}

Alain Goulet, «Pas si simple de refuser l'héritage! Les exemples d'André Gide et de Sylvie Germain »,

Studi Francesi [En ligne], 174 (LVIII | III) | 2014, mis en ligne le 01 novembre 2014, consulté le 17 septembre 2020. URL : http://journals.openedition.org/studifrancesi/1045 ; DOI : https://doi.org/ 10.4000/studifrancesi. 1045

\section{(c) (i) (9)}

Studi Francesi è distribuita con Licenza Creative Commons Attribuzione - Non commerciale - Non opere derivate 4.0 Internazionale. 


\title{
Pas si simple de refuser l'héritage! Les exemples d'André Gide et de Sylvie Germain
}

\begin{abstract}
The examples of André Gide, Sylvie Germain and their characters show to what extent it is futile to try renouncing what one has inherited in order to assert oneself as without a past, having severed links with one's roots and one's culture: inherited traits are a part of one's identity. Gide, who on many occasions would have liked to wipe the slate clean in order to «stand up naked on virgin territory» (Les Nouvelles Nourritures), remained an inheritor, as did his heroes, those of L'Immoraliste, Les Caves du Vatican and Les Faux-Monnayeurs in particular. In the case of Sylvie Germain, the question of inheritance is played out essentially in the depths of the individual's being, in their unconscious and their inner crypt. Thus Le Livre des Nuits and Nuit-d'Ambre constitute a broad drama of cross-generational transmission, a drama which is re-enacted in most of the novels that follow.
\end{abstract}

Naître, c'est aussi automatiquement hériter, car l'héritage est d'abord celui du sang avant de l'être du patrimoine; de la race, des gênes et d'un milieu, avant de se transmettre par l'éducation et la vision du monde, et plus subtilement encore celui d'un inconscient dans lequel se trouve inscrite toute une histoire familiale. Mais chacun est en même temps porteur d'un élan de vie qui lui est personnel, d'une identité à créer et qu'il peut modifier sans cesse, de sorte que certains cherchent à se délester d'un fardeau jugé trop pesant, aimeraient jeter par dessus bord tout le passé, tout héritage, pour se «dresse[r] nu sur la terre vierge, devant le ciel à repeupler» ${ }^{1}$, comme le proclamait Gide dans le feu de sa foi au communisme.

Gide constitue un bel exemple de l'héritier ayant cherché à plusieurs reprises et de différentes façons à larguer les amarres, mais qui, chaque fois, s'est trouvé ramené à lui, à sa condition, à son «idiosyncrasie» comme il aimait le dire. C'est ce que nous verrons d'abord, avant de passer à un autre exemple, celui de personnages de Sylvie Germain chez lesquels on voit bien à quel point l'héritage concerne bien plus l'être que l'avoir, intimement, et chez qui l'héritage colle à la peau et ne peut que refluer en soi, à son insu.

Avec André Gide, considérons d'abord l'héritage sur le plan culturel. Car c'est en héritier qu'il est entré dans sa carrière d'écrivain, et en rentier, en héritier non seulement d'une grande famille bourgeoise mais aussi d'une vaste culture, désireux de s'inscrire à la suite d'une pléiade de quelques grands aînés qu'il admire tels que Flaubert, Barrès, Mallarmé ou Goethe. Mais assez vite, dès son premier voyage en Afrique du Nord, il s'est mis à renier son héritage et à vouloir jeter par dessus bord

(1) Les Nouvelles Nourritures, dans GIDE, Romans et récits. Euvres lyriques et dramatiques,
Gallimard, «Bibliothèque de la Pléiade», 2009, t. 2, p. 751. 
tous les écrivains qui l'ont précédé, toute la culture dont il s'est nourri jusqu'alors, afin d'être vraiment lui-même et de pouvoir exprimer sa nouvelle ferveur, de se livrer au culte des sensations, des nourritures terrestres, et de chanter sa soif de sensations vives et de plaisirs.

Ainsi, dans la sotie Paludes (1895), son héros élabore un «menu type» pour la scène $\mathrm{du}$ «banquet», façon plaisante de proposer d'évacuer après digestion quelques grands noms de contemporains que Gide avait admirés et qui l'ont accompagné lors de ses débuts littéraires, d'écrivains désormais assimilés à de vulgaires cuisiniers dont on se détourne après consommation:

$1^{\circ}$ Potage (selon Monsieur Huysmans).

$2^{\circ}$ Beefsteck (selon Monsieur Barrès).

$3^{\circ}$ Choix de légumes (selon Monsieur Gabriel Trarieux ${ }^{2}$ ).

$4^{\circ}$ Bonbonne d'eau d'Evian (selon Monsieur Mallarmé).

$5^{\circ}$ Chartreuse vert dorée (selon Monsieur Oscar Wilde) ${ }^{3}$.

De fait, les lectures d'À rebours de Huysmans et du Culte du Moi de Barrès avaient conduit Gide à Mallarmé, à Trarieux et à Wilde, au monde littéraire parisien contemporain qu'il entendait à présent jeter symboliquement par dessus bord. «Il faut, Nathanaël, que tu brûles en toi tous les livres», déclarera-il bientôt après, dans ses Nourritures terrestres qu'il ouvre avec une «RONDE/pour adorer ce que j'ai brûlé» qu'il ponctue par l'exclamation: «Nathanaël, quand aurons-nous brûlé tous les livres!» ${ }^{4}$. Gide entend donc répudier tout héritage, désireux d'acquérir désormais toute connaissance par la voie des sensations personnelles, d'expériences propres, décrétant: «Toute connaissance que n'a pas précédée une sensation m'est inutile»5. Qu'il est loin André Walter, son premier héros, son double, qui faisait vœu de vivre dans une «cellule» où il lirait «la Bible, les Védas, Dante, Spinoza, Rabelais, les Stoïques» ${ }^{6}$, et qui entrelaçait ses notes d'une foule de citations! Déjà, le héros du Voyage d'Urien entendait quitter les livres ${ }^{7}$ et, exaspéré par celui qu'Ellis tenait à la main, l'avait jeté à l'eau'.

Mais il n'est pas si simple de renier son héritage, de faire table rase de son passé pour que naisse un être nouveau, et en dépit de toutes ses résolutions en ce sens, de ses tentatives, Gide est resté tributaire d'un héritage qui lui collait à la peau et de sa culture. Et quand, fort de sa foi dans le communisme, il s'écrie triomphant, comme nous l'avons dit: «Table rase. J'ai tout balayé. C'en est fait! Je me dresse nu sur la terre vierge, devant le ciel à repeupler»" ${ }^{9}$, il ne peut s'empêcher de se référer en même temps, dans ses Nouvelles Nourritures, à tout son héritage culturel, à l'Évangile, au Christ et à divers passages de la Bible, comme aussi à la fable grecque, à Goethe, à Francis Jammes, Virgile, Bach, Mozart, Musset...

Ce thème du reniement de l'héritage avait auparavant fait l'objet de plusieurs de ses expérimentations romanesques qui aboutissaient toutes au risque de catastrophe. Ainsi le héros de L'Immoraliste finissait par reconnaître: «Savoir se libérer n'est rien; l'ardu, c'est savoir être libre» ${ }^{10}$. C'est ce qu'illustre le récit qui nous pré-

(2) C'est chez Gabriel Trarieux, «fils du ministre de la Justice et futur auteur dramatique» que Gide a été présenté, le $1^{\text {er }}$ mai 1891 , à Marcel Proust (Voir Claude Martin, André Gide ou la vocation du bonbeur, t. I, Fayard, 1998, p. 144). Trarieux a publié à l'âge de 20 ans son premier recueil de vers (Confiteor: Les Arabesques, La Ritournelle des amoureux, Les Vestales, Révélation de Saint-Jean, le théologien, 1891).

(3) Paludes, dans GIDE, Romans et récits cit., t. 1, pp. 283-284.
(4) Les Nourritures terrestres, ibid., pp. 359-361.

(5) Ibid., p. 361.

(6) Les Cabiers d'André Walter, dans GIDE, Romans et récits. Euvres lyriques et dramatiques cit., t. 1, p. 22.

(7) Le Voyage d'Urien, ibid., p. 186.

(8) Ibid., p. 210.

(9) Les Nouvelles Nourritures cit., t. 2, p. 751

(10) L'Immoraliste, dans GIDE, Romans et récits cit., t. 1, p. 597. 
sente un homme élevé en serre chaude par ses parents, et qui arrive à vingt-cinq ans armé d'une érudition impressionnante - connaissant le latin, le grec, «l'hébreu, le sanscrit, et enfin le persan et l'arabe»-, mais «n'ayant presque rien regardé que des ruines ou des livres, et ne connaissant rien de la vie» ${ }^{11}$. Il tombe gravement malade lors de son voyage de noces en Afrique du nord, reprend goût à la vie à la vue d'un jeune Arabe, et s'opère alors en lui une métamorphose qui le détourne de son être ancien et l'oriente vers la sensation et la vie: «seule ma guérison devait devenir mon étude» ${ }^{12}$, estime-t-il. Et bientôt:

J'en vins à mépriser en moi cette science qui d'abord faisait mon orgueil [...]. Je me découvrais autre et j'existais, ô joie! en dehors d'elles. [...]

Ce fut dès lors celui que je prétendis découvrir: l'être authentique, le «vieil homme», celui dont ne voulait plus l'Evangile[...]. Je méprisai dès lors cet être secondaire, appris, que l'instruction avait dessiné par-dessus. [...]

Et je me comparais aux palimpsestes; je goûtais la joie du savant, qui, sous les écritures plus récentes, découvre sur un même papier, un texte très ancien infiniment plus précieux. [...] Je me livrai voluptueusement à moi-même, aux choses, au tout, qui me parut divin ${ }^{13}$.

Ce reniement de tout le passé qui a fondé son être jusque là, de tout son héritage, le conduit à une série de catastrophes qui font boule de neige: il saborde peu à peu la saine exploitation de son domaine normand, braconne sur ses propres terres; et surtout, tout à sa propre exaltation de la vie, il néglige sa femme dont la santé décline à mesure que la sienne se rétablit et s'exalte, et qui finit par perdre l'enfant qu'elle portait. Après la fausse couche survient une phlébite, puis une embolie, et Michel se détourne d'elle comme d'une «chose abîmée» ${ }^{14}$. Inéluctablement, son comportement conduit sa femme à la mort sans qu'il en prenne conscience, à cause de son «espèce d'entêtement dans le pire» ${ }^{15}$. A la suite de quoi il demeure prostré, hors de tout projet, sans volonté: il n'est plus que l'épave de l'homme éminent qu'il était.

Un autre cas bien symptomatique d'un tel naufrage est celui de Lafcadio, dans Les Caves du Vatican. Le jeune homme s'est trouvé conçu précisément pour incarner un être expérimental en rupture de la continuité familiale, comme un bâtard exclu de toute reconnaissance qui lui donnerait droit à un héritage, comme un être neuf qui pourrait s'inventer hors du poids du passé. «Je tenais [...] à ce que la force de Lafcadio demeurât libre, et sa vertu non asservie par le besoin ${ }^{16}$, déclare son père, Juste-Agénor. Son éducation consiste à l'armer intellectuellement et physiquement, à lui donner le goût du jeu, et à se sentir libre d'accomplir ce que bon lui semble, hors de toute règle et de toute loi. Privé de tout Surmoi, il est ainsi conduit, alors qu'il entend fuir «vers un nouveau monde» pour «supputer les ressources d'une possible humanitée ${ }^{17}$, à commettre son fameux «acte gratuit» consistant à jeter en bas d'un train en marche, par jeu, un vieillard inconnu qui a le malheur de lui déplaire, sans aucune autre raison que de se prouver de quoi il pourrait être capable:

«Un crime immotivé, continuait Lafcadio: quel embarras pour la police! [...] Ce n'est pas tant des événements que j'ai curiosité, que de moi-même. Tel se croit capable de tout, qui, devant que d'agir, recule... Qu'il y a loin, entre l'imagination et le fait!... Et pas plus le droit de reprendre son coup qu'aux échecs» ${ }^{18}$.

(11) Ibid, pp. 598-599.

(12) Ibid., p. 608.

(13) Ibid., pp. 621-622.

(14) Ibid., p. 660.

(15) Ibid., p. 686.
(16) Les Caves du Vatican, dans GIDE, Romans et récits cit., t. 1, p. 1190 (Chapitre supprimé: II, 6).

(17) Les Caves du Vatican cit., p. 1129.

(18) Ibid., p. 1134. 
Après quoi il est tout étonné de se retrouver criminel.

Un autre héros gidien montre aussi qu'il n'est pas si simple de se débarrasser de son héritage. Bernard, dans Les Faux-Monnayeurs, découvrant par hasard sa bâtardise, décide de rompre complètement avec son passé, sa famille, et de partir à l'aventure: «Ne retenons de ceci que la délivrance» ${ }^{19}$. Mais s'il renie un temps sa famille et d'abord son père putatif et son nom, il est incapable de renier sa culture et il se repaît de références culturelles et de citations qui le guident dans sa conduite

Si vous saviez ce que c'est enrageant d'avoir dans la tête des tas de phrases de grands auteurs, qui viennent irrésistiblement sur vos lèvres quand on veut exprimer un sentiment sincère $^{20}$.

reconnât-il. Et après bien des péripéties qui l'ont mûri et fait réfléchir, il remet en cause sa révolte et décide de retourner chez son père.

Dans sa vie, Gide aussi a souhaité de se débarrasser d'une bonne partie de l'héritage reçu de ses parents: en se détournant de son protestantisme et de la Bible, en 1993; en chantant l'évangile de Ménalque dans ses Nourritures terrestres:

Familles, je vous hais! foyers clos; portes refermées; possessions jalouses du bonheur.

[...] me séparer de tout ce qui ne m'est pas indispensable. [...] Ames jamais suffisamment dénuées pour être enfin suffisamment emplies d'amour - d'amour, d'attente et d'espérance, qui sont nos seules vraies possessions ${ }^{21}$.

En prônant qu'il s'agit toujours de se réinventer et d'inventer son avenir:

Le présent serait plein de tous les avenirs, si le passé n'y projetait déjà une histoire. [...]

Nathanaël, ne cherche pas, dans l'avenir, à retrouver jamais le passé ${ }^{22}$.

en vendant son château de La Roque et une partie de ses terres en 1900; en acceptant son homosexualité et en la revendiquant, contre le puritanisme de ses parents et de ses ancêtres... En 1931, il s'écrit même: «La religion et la famille sont les deux pires ennemis du progrès», raison essentielle qui l'amène à adhérer à la cause de la révolution russe qui prétend les abolir pour créer l'homme nouveau.

Mais avec l'âge, voilà qu'il s'attache à l'héritage que constitue le fait d'être Français et de s'en trouver dépossédé: «Où sont ces qualités, ces vertus, qui me faisaient aimer ma patrie? ${ }^{23}$.

De façon plus générale, de quel héritage culturel riche et divers se réclame continuellement son Journal! Il ne cesse d'y souligner l'importance et la richesse du patrimoine de la France ${ }^{24}$, tout en professant: «Il n'y a culture que dans une continuation, et je tiens pour néfastes certains reniements de notre passé» ${ }^{25}$.

Avec Sylvie Germain, la question de l'héritage prend un tour plus aigu, plus dramatique, tragique parfois, surtout dans les premiers romans où la scène secrète se joue dans l'inconscient.

(19) Les Faux-Monnayeurs, dans GIDE, Romans et récits cit., t. 2, p. 175.

(20) Ibid., p. 322.

(21) Les Nourritures terrestres, dans GIDE, Romans et récits cit., t. 1, pp. 382, 400.
(22) Ibid., p. 355 et p. 365.

(23) Journal II: 1926-1950, Paris, Gallimard, «Bibl. de la Pléiade», 1997, p. 769 (5 juillet 1941).

(24) Ibid., pp. 792-795.

(25) Ibid., p. 776. 
Afin de bien considérer la nature et l'action de l'héritage inconscient qui se loge aux tréfonds de l'être, commençons par exposer en quelques mots la théorie de la crypte et du fantôme due aux psychanalystes Nicolas Abraham et Maria Torok, qui concerne aussi l'héritage transgénérationnel ${ }^{26}$. Tout part du concept d'incorporation (opposé à celui d'introjection), phénomène consistant à installer en soi, à la suite d'un trauma ou d'un deuil impossible à élaborer, l'objet prohibé et refoulé, et à l'engloutir en soi comme un corps étranger inassimilable, ce qui aliène le Moi:

Tous les mots qui n'auront pu être dits, toutes les scènes qui n'auront pu être remémorées, toutes les larmes qui n'auront pu être versées, seront avalées, en même temps que le traumatisme, cause de la perte. Avalés et mis en conserve. Le deuil indicible installe à l'intérieur du sujet un caveau secret. Dans la crypte repose, vivant, reconstitué à partir de souvenirs de mots, d'images et d'affects, le corrélat objectal de la perte, en tant que personne complète [...], ainsi que les moments traumatiques [...] qui avaient rendu l'introjection impraticable. Il s'est créé ainsi tout un monde fantasmatique inconscient qui mène une vie séparée et occulte. ${ }^{27}$

Or cette «crypte», «enclave qui transforme le sujet en «gardien de cimetière» ${ }^{28}$, bloque ou parasite son développement, et constitue un noyau traumatique qui ne lui appartient pas, se manifeste par un «fantôme» qui le hante «en lui faisant des signes étranges et incompréhensibles, en l'obligeant à accomplir des actes insolites, en lui infligeant des sensations inattendues ${ }^{29}$. Et elle peut se transmettre de générations en générations, finissant parfois par s'imposer de façon péremptoire trois ou quatre générations plus tard ${ }^{30}$. Alors l'enfant investi de faits passés vécus par un aïeul tend à les revivre au présent. Par exemple, Marie-Laure Aubignat, dans une étude portant sur les répercussions psychopathologiques de la Seconde Guerre mondiale sur trois générations, a mis en évidence une «identification endoscopique, [...] par les petits enfants de survivants, envers l'aïeul survivant, absorb[ant] toute l'énergie familiale autour de l'objet transgénérationnel obsédant, objet qui doit rester clivé ou forclos» ${ }^{31}$.

Tout le premier massif romanesque de Sylvie Germain formé par Le Livre des Nuits (1985) et Nuit-d'Ambre (1987) repose ainsi sur la transmission transgénérationnelle de tout un héritage inconscient dont apparaissent divers signes physiques à chaque génération (couleur des yeux, tache lie-de-vin... ${ }^{32}$ ) et qui, pour l'essentiel, se condense sous la forme d'un cri. Au terme d'une histoire familiale complexe apparaît Charles-Victor, dit Nuit-d'Ambre, qui se trouve investi à l'âge de cinq ans par le cri transgénérationnel poussé par sa mère et qui, par la suite, tente de façon résolue à plusieurs reprises de tourner résolument le dos à sa famille, à son histoire, à tout ce qu'elle peut véhiculer d'héritage, un héritage qu'il devine empoisonné. Son frère a été tué, sa mère en devient folle et meurt, son père est gagné à son tour par une curieuse folie et devient «Fou-d'Elle» jusqu'à en mourir.

(26) Voir N. Abraham et M. Torok, Cryptonymie. Le Verbier de l'bomme aux loups, précédé de Fors, par Jacques Derrida, Flammarion (1976), 1999; L'Écorce et le noyau, Aubier Flammarion, 1978; et C. Nachin, La Méthode psychanalytique. Évolutions et pratiques, Armand Colin, 2004, pp. 129-160.

(27) L'Écorce et le noyau cit., p. 266.

(28) Ibid., pp. 254-255.

(29) Ibid., p. 266.

(30) Voir Aie, mes aïeux!, d'A. A. SCHÜTZENBERGER (Desclée de Brouwer, 1993), et Comment paye- $t$ -on les fautes de ses ancêtres (Desclée De Brouwer, 1998).
(31) M.-L. Aubignat, Répercussions psychopathologiques de la seconde guerre mondiale sur la troisième génération, «Le Journal international de victimologie», n. 14, mars 2007.

(32) Ainsi pour Rose-Héloïse et Violette-Honorine: «leur héritage était double, non seulement parce qu'elles avaient reçu la couleur des yeux de leur mère et ses beaux cheveux frisés, mais surtout parce qu'elles affichaient à la tempe gauche une tache lie-de-vin»; «Marie n'enfanta pas des têtards mais deux fils qui n'avaient pas d'autre particularité que celle reçue par tous les enfants Péniel en héritage». (Le Livre des Nuits, Gallimard, «Folio», p. 135,220$)$. 
«Voilà, dit Nuit-d'Ambre à la mort de son père, tout est dorénavant fini du temps du frère. [...] Il a emporté la mère, puis le père à sa suite. Mais nous, il ne nous aura pas. Nous n'avons rien à voir avec lui, plus rien à faire avec eux» [...].

Il se voulait un adolescent sans mémoire, sans attache ni nostalgie ${ }^{33}$.

Mais il se trompe, et il est bientôt rattrapé par l'héritage familial et la mémoire du passé. Sa première rencontre à Paris où il est parti est celle d'un étudiant qui lui parle de la ratonnade des manifestants algériens d'octobre 1961. Et Nuit-d'Ambre de protester avec véhémence:

«Je m'en fous! Je me fous de tous vos morts, de tous les morts, de toutes les guerres. Je me fous de mes propres morts! Et puis je suis de l'après-guerre, moi, d'après toutes les guerres! Je me fous de l'Histoire. Je me fous pas mal d'être français ${ }^{34}$.

Sa rencontre la plus troublante sera celle d'une vieille femme qui sollicite la charité en vendant des citrons pourris. Elle l'entraîne malgré lui dans un parcours fantastique qui s'avère être un voyage dans ce passé qu'il s'est acharné à fuir. Il retrouve l'antre dans lequel il s'était réfugié étant enfant, après la mort de son frère, avec «l'épave d'un cabinet» dont il avait fait son royaume. Et soudain, sa mémoire revient: «Nuit-d'Ambre se revit dans les bas-fonds de son enfance de sauvage; les entrepôts de l'usine désaffectée, le vieux bunker au fond des bois, les latrines à la porte branlante percée d'une lucarne en forme de cœur. Hauts lieux de sa colère d'enfant rebelle» ${ }^{35}$.

Alors, pour tuer sa mémoire qui s'obstine à ressurgir contre son gré, il décide de commettre l'irréparable en tuant celui qu'il était sous la forme de son double: un petit mitron qui quête son amitié et qu'il attire dans un guet-apens. Mais son crime ne fait que le ramener à lui-même et à son passé. Au moment de la mise à mort, dans les yeux de sa victime «devenus miroirs, il aperçut le reflet de son propre visage». De fait, Nuit-d'Ambre est amené peu à peu à renouer avec son passé, à retrouver sa jeunesse qu'il «avait passé la nuit à [...] vomir» ${ }^{36}$. Il retourne sur la terre de son enfance, où le fils qu'il a conçu après son crime le conduit au surgissement du passé refoulé, jusqu'au «cri de sa mère [qui] l'avait irrémédiablement déchiré». Il tente alors de se suicider, mais il en est arrêté par une lutte avec l'ange qui lui fait retrouver «son regard du dedans $\rangle^{37}$.

Ainsi, tout cet univers se trouve-t-il placé sous l'égide des Nuits, nuits de la transmission de la mémoire des ancêtres, de leur crypte enfouie en eux, que chacun cherche à fuir, à oublier, mais dont ils ne parviennent jamais à se délivrer. Car

La nuit renversait sans cesse son encrier dans la mémoire des hommes et la mémoire bruissait continûment du très confus murmure tissé par le temps s'écrivant, par le passé s'enlaçant au présent, s'élançant vers l'avenir. La nuit dictait obstinément aux hommes les dits de la mémoire, de leur propre mémoire qu'ils tentaient cependant toujours de fuir, de faire taire, de renier. La nuit, tenace, forçait les hommes à faire mémoire. A faire mémoire jusque dans les déserts de l'oubli. Car cette encre de nuit était autant d'oubli que de mémoire.

Et la mémoire filait en chacun, toujours plus vite, toujours plus loin, au fil de l'âge. [...] La mémoire en eux avait recouvré tous ses droits, repoussé ses frontières par-delà les limites de la peur, de la douleur et de la honte. La mémoire en eux avait retrouvé ampleur et mouvement, et était devenue gravide. Elle portait bien davantage que les souvenirs des autres; elle portait traces d'eux, des traces vives qui étaient forces bien plus que formes. Des traces en marche ${ }^{38}$.

(33) Nuit-d'Ambre, Gallimard, «Folio», p. 134.

(34) Ibid., p. 184.

(35) Ibid., p. 244.
(36) Ibid., p. 292 et p. 318.

(37) Ibid., p. 401 et p. 403.

(38) Ibid., pp. 414-415. 
Tel avait été particulièrement le cas pour Nuit-d'Ambre:

La nuit en lui avait glissé comme une eau lourde et violente. [...] La nuit en lui avait roulé le sourd mugissement des houles noires de la mémoire. [...] Mais Nuit-d'Ambre-Vent-de-Feu n'avait cessé de résister, de crier non, de dresser les brisants de ses cris contre ces déferlements de mémoire.

Inéluctablement, la mémoire de l'héritage en lui a été la plus forte, triomphant de toutes ses manœuvres et de tous ses reniements. En définitive,

le chancre de son soleil factice n'avait plus cessé de s'ouvrir, de saigner, de craquer, de suer. Plaie à métamorphoses, allant toujours en s'enfiévrant. Plaie qui longtemps s'était boursouflée, défigurée. Plaie refrappée par la main d'un inconnu venu lutter avec lui, au corps à corps. Depuis ce jour la plaie s'était à nouveau transformée; elle se transfigurait. Elle s'ouvrait toujours, et saignait et brûlait, mais autrement. Elle saignait moins qu'elle ne pleurait des larmes de sang, et sa sueur aussi était de larmes. A la place du soleil factice transsudait un visage. La plaie devenait suaire ${ }^{39}$.

Dans L'Enfant Méduse, la brutale rupture d'héritage s'opère à la suite du viol incestueux dont est victime pendant trois ans la petite Lucie, viol commis par son grand frère, son demi-frère en fait. Or il se trouve que celui-ci avait été à son insu investi par leur mère de tout un héritage mortifère, se trouvant consacré comme l'incarnation de son père mort à la guerre, ce qui s'est mis à brouiller toutes les frontières des relations familiales:

Son corps, - tombeau vivant. Ferdinand a grandi sous le regard vigilant de sa mère ainsi que s'édifie un mausolée précieux sous la surveillance des fidèles qui en ont établi la commande afin de célébrer, selon leur goût très strict et dévotieux, la mémoire d'un défunt vénéré. Ferdinand a grandi sous le regard de veuve de sa mère, comme s'élève un mémorial. Il a grandi, seul, sous ce regard étincelant qui tout à la fois mendiait et exigeait de lui une absolue ressemblance avec l'époux mort à la guerre ${ }^{40}$.

En conséquence et comme sous le faix d'un enchantement, le beau Ferdinand est devenu violeur de fillettes, et il se met à abuser de sa petite sœur qui se trouve à sa merci. Alors, Lucie rompt tout lien avec ce passé pervers et avec le monde, «elle se veut seule [...], elle a instauré le vide autour d'elle» ${ }^{41}$. Elle ne veut surtout plus rien avoir à faire avec sa mère qui «est en même temps celle du loup et celle de la chèvre. [...] Sa mère, une louve presque. Une traître qui s'ignore» ${ }^{42}$. Son unique compagnie devient celle de la faune des marais et des bois:

Sa préférence va aux insectes, aux batraciens, aux reptiles et aux oiseaux. Tout ce qui rampe et ce qui vole, tout ce qui saute par bonds vifs; [...] bêtes à métamorphoses, à mues et à camouflages ${ }^{43}$.

Cette échappée de l'héritage familial, social et humain aiguise sa volonté de vengeance. Son frère meurt et elle «se veut, et se sait, l'assassin de son frère» ${ }^{44}$. Mais elle paie cher ce secret, et demeurera en marge de la vie. Plus tard, «Lucie avait hérité d'une certaine somme qu'elle s'était empressée de dépenser. Elle était partie arpenter

(39) Ibid.,pp. 415-417.

(40) L'Enfant Méduse, Gallimard, Folio, p. 78.

(41) Ibid., p. 92.
(42) Ibid., p. 98.

(43) Ibid., p. 127.

(44) Ibid., p. 250. 
le globe en tous sens» ${ }^{45}$. Elle demeure en compagnie de ses voix familières, à l'écoute du «chant de la terre, et celui des marais, celui du vent, et celui des forêts», dans «un vide immense, depuis l'enfance ${ }^{46}$. Là encore, l'abolition de l'héritage s'avère destructeur de l'être.

Mais il advient qu'à partir de La Pleurante des rues de Prague (1992), l'œuvre de Sylvie Germain bascule. Auparavant, nous avions affaire à des personnages encombrés à leur insu par un héritage toxique et mortifère qu'ils portent en eux. Or, la figure centrale de La Pleurante des rues de Prague est une figure fantastique, une «géante au corps lourd de la mémoire des hommes» ${ }^{47}$, incarnation de la bonne mère universelle qui se serait chargée de l'héritage des douleurs et des deuils injustes et violents, et qui s'est faite l'héritière de toute la misère humaine, la consolatrice de toutes les victimes des violences de l'Histoire.

Les héros des romans qui suivent La Pleurante des rues de Prague s'emploient donc, eux, à se réapproprier lentement un héritage dont ils ont été coupés. Ainsi, dans Eclats de sel, Ludvik retrouve-t-il le sens de sa vie qui s'est enlisée grâce à un de ses cahiers qu'il avait jadis offert à son maître Joachym Brum, cahier qui, soudain, «lui restitu[e] son passé, sa mémoire. Lui redress[e] le cœur, l'esprit» ${ }^{48}$, et le rend à lui-même. Dans Tobie des marais, la vieille Déborah s'acharne à maintenir tout un héritage familial rompu par l'exil, et elle se fait la gardienne de toute la tradition yiddish, soucieuse en particulier de préserver la mémoire des morts sans sépulture. Son chant est «une houle montée de l'autre côté du siècle, et de plus loin encore, une clameur d'étoiles dans la nuit, [...] - et tous ses morts étaient pour elle demeurés des

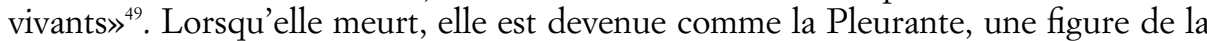
compassion pour tous les morts de sa famille qui l'ont précédée $e^{50}$.

Ensuite apparaissent des héros privés de tout héritage: Laudes-Marie, l'héroïne de Chanson des mal-aimants (2002), a été abandonnée à sa naissance et passe sa vie à espérer connaître un jour sa mère qu'elle entrevoit dans des visions. Comme elle le dit avec humour, «mon arbre généalogique est un bonzaï tout ébranché, cul-de-jatte côté racines», et ses parents sont «deux renégats qui [lui ont] légué le tourment de leur anonymat pour tout héritage $\gg^{51}$. Toute son existence se présente comme une longue marche solitaire vers un sourire maternel qu'elle finit par apercevoir à la fin du roman, un «sourire de délivrance» ${ }^{52}$.

Quant à Magnus, il s'est trouvé amnésique, coupé de tout passé et de tout héritage à l'âge de cinq ans, à la suite des bombardements de Hambourg qui ont transformé celle qui devait être sa mère en torche humaine. Cependant, l'amnésie ne peut être complète et on ne se trouve jamais coupé complètement de son héritage, car «l'immémorial est pailleté de traces, infimes et têtues»" Mais une des conséquences de cette rupture avec soi, c'est que Magnus se trouve d'abord privé de «structuration affective», et pendant longtemps, «il ne sait pas comment aimer ${ }^{54}$. Lorsqu'il a vingt ans, il se rend au Mexique, espérant y trouver des traces de celui qu'il pense être son père, mais c'est alors qu'il est pris d'une anamnèse qui lui fait revivre l'événement de juillet 1943 qui l'a privé de mémoire:

(45) Ibid., p. 266.

(46) Ibid., p. 270 et 272.

(47) La Pleurante des rues de Prague, p. 103.

(48) Éclats de sel, p. 159.

(49) Tobie des marais, p. 114.

(50) Cf. «Déborah Rozmaryn, née Rosenkranz, fut enterrée au cimetière la veille du jour où elle aurait dû fêter ses cent ans. [...] Dans les jours qui suivirent l'inhumation de Déborah il se passa un phénomène étrange; de l'eau se mit à sourdre de la terre autour de la tombe. [...] La source n'était autre que Déborah dont le corps exsudait une à une les larmes qu'elle avait si longtemps retenues; le corps pleurait, se délivrait d'un chagrin démesuré, il s'épurait, se lavait dans l'aveu de sa peine». (Ibid., p. 119-120).

(51) Chanson des mal-aimants, p. 14 et p. 35.

(52) Ibid., p. 245.

(53) Magnus, Albin Michel, 2005, p. 11.

(54) Ibid., p. 74. 
le sursaut de mémoire qui lui vient monte d'ailleurs, de plus loin, c'est une lame de fond s'élançant du cœur de la nuit où il est mort, lui, Adam Schmalker, avant qu'il ne se fasse appeler ainsi, et avant même qu'il ne se soit nommé Franz-Georg Dunkeltal.

Par sa vision, une porte s'est ouverte vers son passé, et dans le délire qui suit, on l'entend proférer «des phrases dans une autre langue» que l'allemand censé être sa langue maternelle, qu'on se trouve «incapable d'identifier ${ }^{55}$. Ainsi découvre-t-il que «notre mémoire [procède à] un ressassement continu, mais si bas, si confus, comme celui du sang dans nos veines, qu'on ne l'entend pas» ${ }^{56}$. A partir de ce premier reflux du passé, il apprend à aimer, et par la piste de la langue surgie pendant son délire, on devine que sa prime enfance a dû être islandaise. Plus tard, il aura l'intuition de son vrai nom, un «nom qui lui est totalement étranger ${ }^{57}$, mais à son réveil, il est incapable de le retrouver, et l'accès à son héritage lui restera à jamais interdit. Alors, cessant de scruter le passé qui lui échappe, il comprend qu'il a la liberté d'inventer son avenir, et «part au-devant de son nom qui toujours le précède» ${ }^{58}$.

Dans Magnus, plus encore que dans tous les autres romans de Sylvie Germain, se multiplient parallèlement les traces d'un héritage culturel de l'auteur, riche et varié, comme pour compenser l'amnésie de son héros, coupé de ses racines. En effet, ses différents chapitres sont ponctués de notes, séquences et fragments qui déploient toutes sortes de références et de citations: s'y succèdent un chant de Schubert, un article sur Friedrichshafen, Dietrich Bonhoeffer, Paul Celan, l'ours, le bélier, Juan Rulfo, Fabienne Bradu, l'opération «Gomorrah», etc., autant de traces qui attestent la façon dont Sylvie Germain se sent portée par une culture qui l'imprègne et nourrit son imaginaire.

L'Inaperçu (2008) enfin présente une arborescence ${ }^{59}$ familiale qui oscille entre le gardien étroit des traditions, Charlam Bérynx, au «double prénom hérité de ses deux grands-pères» ${ }^{60}$, et Céleste Bergance qui, délaissée par son mari, conçoit une bâtarde avec un Allemand pendant la guerre et se sent délivrée de «l'état de soumission à l'ordre imposé par son entourage, d'obéissance à la loi tacite des bienséances qui se nourrit de tant de mensonges, de mystifications ${ }^{61}{ }^{6}$, mais qui s'en trouvera bientôt châtiée. Elle a nommé sa fille Zélie, ce qui lui vaut «une réprobation accrue de la part des membres de sa famille [... qui estime] que le nouveau-né, marqué d'une double tare dès l'origine, la bâtardise et le déshonneur patriotique, était indigne d'hériter du prénom d'une aïeule que tous avaient respectée et aimée» ${ }^{62}$. On voit donc combien le nom et le prénom sont un concentré symbolique très marqué de l'héritage familial.

Zélie, qui oscille entre ses deux pères, l'un putatif et l'autre fantôme, se met d'abord en quête de son héritage, fugue, puis renonce à connaître son vrai père responsable de la souffrance et de la mort de sa mère. Mais le traumatisme de la rupture de l'héritage familial la conduit à l'internement dans un établissement psychiatrique où elle finit par se suicider de désespoir. Ainsi, une nouvelle fois, s'est joué un de ces drames obscurs auxquels peut conduire la rupture avec l'héritage familial et social.

Pierre, le frère de Zélie, voit lui aussi sa vie pervertie et tordue à cause de cette rupture aux conséquences désastreuses. Il connaît une sorte de descente aux enfers qui lui permet «d'aller à la rencontre de ses morts et de faire la paix avec eux» ${ }^{63}$, puis de s'extraire de sa retraite pour tenter de renouer avec les deux femmes qui pourraient l'aider à se réinsérer dans le temps de sa vie et dans ses affections, mais il échoue: la

(55) Ibid., p. 107.

(56) Ibid.

(57) Ibid., p. 248.

(58) Ibid., p. 274.

(59) Le premier titre de L'Inaperçu était Arbores-
(60) L'Inaperçu, Paris, Albin Michel, 2008, p. 18.

(61) Ibid., p. 252.

(62) Ibid., p. 251.

(63) Ibid., p. 292. cences. 
dernière scène du roman oppose précisément Charlam, le gardien de la tradition et de la transmission familiale, à Pierre, rejeté, désancré, condamné à l'errance.

Après tant d'autres, Sylvie Germain nous indique donc bien que l'héritage essentiel, celui qui compte vraiment et qui agit à notre insu, s'inscrit non pas comme avoir, mais comme être. C'est ce qu'elle manifeste particulièrement bien dans son dernier ouvrage, Le Monde sans vous, qui est un tombeau consacré à sa mère et à son père:

En héritage, un souci d'or: porter le poids de ton absence, porter le poids des soleils en éclipses, des hiers révolus. [...] Source et creuset de ma vie — ma mère. [...] Ma mère, mon enfant inversé. [...] Des traces subsistent, confuses et coriaces. [...] Tout est palimpseste ${ }^{64}$. [...] Le monde mugit de l'incessant dialogue de sourds échangé entre les vivants et les morts ${ }^{65}$.

écrit-elle à propos de sa mère. Et le cimetière où reposent ses parents se fait «livre dispersé dans l'immobilité, le silence» ${ }^{66}$, tandis que leurs corps et leurs souvenirs sont intégrés à soi, ce qui la transforme en passeur après eux:

Corps des siens qui ne sont plus, entrés dans la dérive du passé. Corps surtout de ses parents, devenus dans la vieillesse comme un unique corps arraché progressivement au mouvement, au regard, au langage [...]. C'est son tour de prendre la place d'amont, et de veiller sur la mémoire [...]. Son tour est venu de devenir passeur. / Passeur d'enfance et de mémoire, passeur de mots et de regard ${ }^{67}$.

Car

Le silence qui désormais enveloppe la personne défunte [...] reflue vers nous, il s'insinue dans la langue toujours en cours et en vigueur en nous, il s'infiltre dans nos mots $[\ldots]^{68}$.

Enfin, dans son essai «Acte de mémoire», Sylvie Germain place même la «notion d'héritage [...] au cœur de la conception judéo-chrétienne du monde et de la relation entre Dieu et l'humanité. [...] Elle en est le cœur, le souffle, la dynamique» ${ }^{69}$. Car la Bible présente la Création «comme un don, un legs adressé à l'homme», comme un testament qu'il est de la responsabilité de chacun de respecter et de faire fructifier. Et, ajoute-t-elle,

L'héritage est comme le festin dont parlent les Evangiles: une joie qui s'accroît avec le nombre des invités» ${ }^{70}$.

Accepter ou refuser l'héritage? En fait, l'alternative est toujours biaisée; car ce qui est important dans la question de l'héritage n'est pas tant l'avoir, on l'a vu, que l'être qui se construit toujours à partir de son héritage familial et, au-delà ou en deçà, par notre insertion dans une culture et une spiritualité. Quoi qu'on veuille ou qu'on fasse, chacun est l'héritier d'une histoire, d'un passé, transmis consciemment ou ni-

(64) Ce terme de «palimpseste» prend ici une valeur opposée à celle que lui donnait Gide dans L'Immoraliste (voir supra): pour Sylvie Germain, cette notion souligne l'héritage immémorial dont on est redevable, incite à rechercher une origine lointaine, comme elle l'a fait pour son héros dans Le Livre des Nuits; pour Gide et son immoraliste, le travail du palimpseste consiste à retrouver l'être naturel originel sous les défroques de l'éducation et de la culture.
(65) Le Monde sans vous, Albin Michel, 2011, pp. 13, 31, 32, 36, 37.

(66) Ibid., p. 99

(67) Ibid., pp. 106-107.

(68) Ibid., p. 127.

(69) S. GERMAIN, «Acte de mémoire», in Quatre actes de présence, Paris, Desclée de Brouwer, «Littérature ouverte», 2011, p. 109.

(70) Ibid., p. 113. 
ché dans l'inconscient, et qui n'en agit alors que plus sûrement ${ }^{71}$. On comprend bien que, pour avancer librement dans sa vie et inventer sa voie, il faut que l'héritage ne soit pas trop lourd à porter et qu'on ne s'en sente pas prisonnier. Les exemples pris chez Gide et Sylvie Germain paraissent exemplaires du besoin qu'on peut éprouver de prendre ses distances avec un héritage jugé trop encombrant, mais aussi du fait que celui-ci n'en reste pas moins toujours là, tapi dans l'ombre, au cœur de chacun, conditionnant ceux qui ont pensé s'en débarrasser, les conduisant à leur insu.

Et puis, Sylvie Germain l'indique bien, nous sommes pétris de mots, et ceux-ci manifestent toujours notre héritage intime et profond tel que nous l'avons reçu, fait fructifier, et tel que nous le transmettrons.

On pourrait allonger la liste de bien d'autres exemples puisés dans la littérature française. Qu'on songe par exemple à la famille des Rougon-Macquart à jamais prise dans les mailles de l'héritage familial et la fatalité de l'hérédité, ou simplement à Phèdre, «la fille de Minos et de Pasiphaé» ${ }^{72}$, sur qui pèse donc une hérédité bien chargée entre ses deux pôles opposés du monde d'en bas et du monde d'en haut. De toute façon, comme on le sait, noblesse oblige!

ALAIN GOULET

(71) Voir par exemple: E. GAUBERT, De mémoire de foetus - L'béritage familial s'inscrit dans nos cellules dès la conception, Gap, Le Souffle d'or, 2011;
C. RIVEST, Le Roman familial. L'héritage psychologique, Montréal, Ed. du Cram, 2003.

(72) RaCINe, Phèdre, I, 1, vers 36. 